\title{
Enseñanza de la resolución de problemas matemáticos: Un análisis de correspondencias múltiples
}

\section{Teaching how to solve mathematical problems: An analysis of multiple correspondences}

DOl: https://doi.org/10.32870/dse.v0i21.629

\author{
Elizabeth Donoso Osorio* René \\ Valdés Morales** Prisila \\ Cisternas Núñez*** Pablo \\ Cáceres Serrano****
}

\begin{abstract}
Resumen
Esta investigación se propuso como objetivo general analizar las actividades típicas de aula (ATA) en clases de Resolución de problemas matemáticos con el propósito de identificar el tipo de acciones vinculadas a su enseñanza en nivel primario. Para ello se analizaron las ayudas pedagógicas, las estructuras de participación y el trabajo colaborativo existentes en los momentos de inicio, desarrollo y cierre, llevándose a cabo un análisis de correspondencias múltiples (ACM). Los resultados señalan que las acciones más relevantes se presentan solo en algunos momentos de la clase y que si bien se realizan los problemas propuestos, no se incentiva la comprobación de los resultados, la capacidad argumentativa ni el trabajo colaborativo. Se concluye además, que las ayudas más frecuentes son de bajo impacto en el aprendizaje mediado y que las interacciones entre profesor y alumno no tienden a la retroalimentación.
\end{abstract}

Palabras claves: Enseñanza de las matemáticas - educación matemática - solución de problemas - correspondencias múltiples - educación.

\begin{abstract}
The overall aim of this research is to analyze the typical classroom activities (TCA) in Mathematical Problem Solution classes in order to identify the type of actions linked to their teaching at the primary school level. Pedagogical aids, participation structures and collaborative work done at the beginning, development and closing moments were analyzed through a multiple correspondence analysis (ACM). The results indi-
\end{abstract}

* Doctora en Instrucción y Currículum. Profesora de la Escuela de Pedagogía, Pontificia Universidad Católica de Valparaíso, Chile. Línea de investigación: Aprendizaje escolar. elizabeth.donoso@pucv.cl

** Doctor en Psicología. Profesor de la Escuela de Pedagogía, Pontificia Universidad Católica de Valparaíso, Chile. Línea de investigación: Inclusión educativa. rene.valdes@pucv.cl

** Maestra en Educación. Profesora de la Escuela de Pedagogía, Pontificia Universidad Católica de Valparaíso, Chile. Líneas de investigación: Educación especial, inclusión y evaluación.prisila.cisternas@pucv.cl

**** Doctor en Psicología. Profesor de la Escuela de Psicología, Pontificia Universidad Católica de Valparaíso, Chile. Líneas de investigación son: Modelos psicométricos, modelos de diagnóstico cognitivo y talento académico.. pablo.caceres@pucv.cl 
cate that the most relevant actions take place only in some moments of the class and that, although the problems proposed are worked on, the verification of the results, argumentative skills and collaborative work are not encouraged. We also concluded that the most frequent aids have little impact on mediated learning and that the interactions between teacher and student do not point towards feedback.

Keywords: Teaching mathematics - mathematical education - problem solving - multiple correspondences - education.

\section{Introducción}

La resolución de problemas matemáticos pone en juego no solo procesos cognitivos necesarios para la ejecución de tareas escolares, sino que expresa el capital mismo de las matemáticas. Sin embargo, resolver problemas matemáticos no es un trabajo fácil; comprende una serie de complejidades que no permite a muchos estudiantes alcanzar los aprendizajes curriculares en esa área en particular. Es en ese momento en que uno como docente e investigador debe decidir dónde prestar atención: en el aprendizaje del estudiante o en la enseñanza del profesor, aun cuando sabemos que ambos procesos están interrelacionados. En este artículo apostamos por lo segundo. Consideramos que profundizar en las prácticas docentes centradas en el desarrollo de habilidades matemáticas a través de la resolución de problemas permitirá agudizar la comprensión y apuntar, desde la investigación, a conocer y reducir los factores que dificultan el desempeño en resolución de problemas matemáticos. Cuando hablamos de problemas matemáticos, no nos estamos refiriendo a la mera resolución de ejercicios, sino que nos referimos a aquellas situaciones complejas que poseen un contexto verbal y semántico que requieren el uso de habilidades matemáticas para su ejecución.

Para analizar las prácticas docentes, se utilizó el sistema de análisis de la interacción que acontece en los ciclos conversacionales profesor-alumnos, ampliamente descrito en Sánchez, García, Castellano, Sixte, Bustos y García-Rodicio (2008). En este sistema de análisis, el ciclo es entendido como una unidad comunicativa en la que se lleva a cabo un conjunto de intercambios respecto de procedimientos o contenidos involucrados en una tarea, pregunta, orden, entre otros. Este sistema considera tres dimensiones: 1) el modo en el que se organiza la interacción entre alumnos, profesores y tareas (el cómo); los contenidos instruccionales que se generan durante la interacción y a través de patrones y estructuras de interacción (el qué); y 3 ) el grado de participación en la elaboración del contenido que se hace público (el quién) y las ayudas (mediaciones) utilizadas por el profesor.

A partir de este sistema de análisis, nos propusimos como objetivo general analizar las actividades típicas de aula (ATA) en clases de resolución de problemas matemáticos, con el propósito de identificar el tipo de acciones vinculadas a su enseñanza en nivel el primario. Los objetivos específicos son dos: 1) describir las ayudas pedagógicas y las estructuras de participación pre- 
sentes en las clases de resolución de problemas matemáticos, y 2) caracterizar el trabajo colaborativo entre estudiantes en las clases de resolución de problemas matemáticos.

\section{Resolución de problemas matemáticos}

La resolución de problemas matemático es un proceso que requiere una serie de pasos que se acompañan con la complejidad progresiva de la ejecución por parte de quienes lo resuelven (Callejo, 1996). Por su parte, Polya (1965) explicita estrategias esenciales para la resolución de un problema: comprender el problema, trazar un plan para resolverlo, poner en práctica el plan y comprobar los resultados. Estudios posteriores en que los problemas fueron presentados con una pregunta al inicio, arrojaron evidencias que apoyan que el elemento discursivo favorece la construcción del modelo de la situación (Thevenot, Devidal, Barrouillet y Fayol, 2007), lo que significa que el estudiante representa el problema, integrando información del texto con información o conocimiento del lector que está más allá de dicho texto (Sánchez, 2003). Brissiaud y Sander (2005), por su parte, propusieron un marco que denominan Situation Strategy First, según el cual, ante un problema se construye un modelo de la situación que activaría en el alumno estrategias que, basadas en la situación, proporcionen en un tipo de problema la solución numérica directamente.

Por otro lado, Hino (2007) releva el comportamiento de los estudiantes al resolver problemas en donde el contexto cobra relevancia junto con el desarrollo de las habilidades de resolución de problemas. En este proceso, el docente pasa a ser un actor principal ya que es el agente encargado de capturar su atención desde un comienzo y brindar los apoyos que los alumnos requieran. En este sentido, Lemke (1998) da relevancia a la interacción social que se produce entre el docente y los estudiantes, en una clase que pone en juego el conocimiento disciplinar desde la perspectiva social como fundamento en las estrategias de enseñanza utilizadas.

Desde esta perspectiva, cobra sentido el análisis de las discusiones dialógicas que se dan en las clases. Es así como Cazden (1988) se refiere a la estructura conocida como Iniciación-Respuesta-Evaluación, enfocándose en el tiempo en el que se discute y se desarrollan ideas cuando la interacción se dé en este formato I-R-E, de manera que los estudiantes desarrollan procesos cognitivos elevados que les permiten una mayor comprensión y, con ello, mayores posibilidades de aprendizajes significativos (Copeland, 2004). Lo anterior tiene lugar en la Actividad Típica de Aula (ATA), definida por García, Rosales y Sánchez (2010) como "cada una de las actividades que integran una sesión de clase, como la explicación, la lectura colectiva, la resolución de problemas matemáticos, etc. De esta manera, se puede decir que cada una de las sesiones de una unidad didáctica está compuesta por diferentes ATA" (García, Rosales y Sánchez, 2010: 366).

Las investigaciones corroboran que la interacción en el aula es un proceso que debe ser valorado, analizado y mejorado por parte de los docentes y estudiantes. Los sistemas lingüísticos en esta interacción debiesen considerarse tal como lo realizan autores como Sinclair y 
Coulthard (1975), quienes analizaban el turno e intercambio desde unidades pequeñas a más grandes. Además, los estudios realizados en cuanto a la resolución de problemas en el aula aseguran que existen diferencias entre los docentes en servicio y los docentes en proceso de formación. Los primeros se aseguran que los estudiantes comprendan mediante indagaciónrespuesta-feed back (IRF), mientras que los segundos no desarrollan procesos de interacción que brinden mayores oportunidades de aprendizaje para los estudiantes (Rosales, Orrantia, Vicente y Chamoso, 2008).

En este marco, es importante destacar también los aportes de autores como Orrantia, Múñez, Fernández y Matilla (2012), quienes dan relevancia a la enseñanza directa de la resolución de problemas como estrategia significativa para dicho aprendizaje en los estudiantes. En esta misma línea, Toboso (2005), en un estudio acerca de la resolución de problemas y la incidencia de las habilidades verbales y cognitivas para el proceso de resolución, comprobó que la mayor dificultad de los estudiantes se centra en la comprensión del problema y en el reconocimiento de la naturaleza del mismo que le da significado y permite seleccionar el plan de resolución, así como, organizar las estrategias que ordenan la secuencia de los pasos a seguir. El mismo autor corrobora los resultados de su investigación diez años después, en 2015.

\section{Dificultades de aprendizaje y enseñanza de las matemáticas}

Cuando se estudian las dificultades de aprendizaje en el área matemática y la resolución de problemas matemáticos, es preciso considerar las estrategias que utilizan profesores y estudiantes para dar respuesta a las tareas plateadas; esto nos permite acercarnos a conocer cómo los alumnos reciben e interpretan la información vinculada a esta actividad. El interés en cómo los procesos de enseñanza tienen impacto en el aprendizaje de los estudiantes se ha levantado como uno de los más importantes aspectos a considerar cuando se refiere a las dificultades de aprendizaje (Alvarado, Cabezas, Falck y Ortega, 2012; Araya y Dartnell, 2008; Bravo, Falck, González, Manzi y Peirano, 2008; Manzi, Strasser, San Martín y Contreras, 2008; Lara, Mizala y Repetto, 2010; Olfos, 2010).

En el contexto de la enseñanza de las matemáticas, algunos autores señalan que las dificultades de aprendizaje están estrechamente vinculadas a una enseñanza desprovista de conocimientos específicos de matemáticas que permitan a los estudiantes explorar sus posibilidades de aprendizaje (Tambychik y Mohd Meerah, 2010; Aguilar, Aragón y Navarro, 2015). Las dificultades más presentes serían las siguientes: dificultad para comprender globalmente un problema matemático, dificultades para distinguir y analizar la información útil de un problema, dificultad para organizar una solución viable al problema, dificultades para razonar matemáticamente y dificultades heurísticas o estrategias generales de resolución (Chamorro, 2006; Giménez y Pedro da Ponte, 2004; Alsina y Planas, 2008; Alsina, 2012).

Diólopos sobre Educación 
En el contexto de la pedagogía en general, algunas investigaciones en este ámbito destacan por el análisis que se realiza en el discurso que prevalece en las clases de la asignatura de matemática, en donde se observa de forma recurrente un patrón Indagación-Respuesta-Evaluación (IRE), además, de las actividades propuestas que apuntan a aspectos más bien sociales de los participantes (Sánchez y Coll, 2007).

Por su parte, investigadores como Sánchez, García y Rosales (2010) han indagado acerca de los procesos de interacción sustentados en un enfoque o modelo cognitivo centrado en qué se enseña. Entre sus aportes, se destacan dos elementos fundamentales para nuestro estudio: el primero vinculado con la interacción, la cual es entendida como un conjunto de intercambios comunicativos que se dan entre profesor y estudiante en el desarrollo de una actividad pedagógica en el contexto de la enseñanza y el aprendizaje; el segundo elemento tiene que ver con el conjunto de acciones repetitivas en el tiempo, es decir, con patrones que organizan las actividades desarrolladas entre profesor y estudiante, esto es, las acciones que se dan repetidamente en la acción del profesor, lo que, en nuestro caso, correspondería a las actividades que acontecen en la resolución de problemas matemáticos.

En el caso de Chile, diversos estudios han señalado que las clases de matemáticas se caracterizan por ser bajas en resolución de problemas (Felmer et al., 2014), a pesar que en el sistema educativo chileno se fomenta su desarrollo (Mineduc, 2009). Autores como Preiss, Larraín y Valenzuela (2011), Espinoza, Barbé y Gálvez (2009), y Alfaro y Gormaz (2009) han demostrado que la enseñanza de las matemáticas es débil en cuanto a incorporación de problemas, con pocas habilidades de los estudiantes, generando resultados insatisfactorios para las escuelas y para las pruebas de medición. Las clases serían espacios de ejercitación sin mayor desarrollo de habilidades para su ejecución. Valdés (2015) menciona que los estudiantes enfrentan el $5^{\circ}$ año de primaria con disminuida capacidad para comprender un problema y, por lo tanto, para resolverlo.

Para este estudio, es importante centrarnos en lo que actualmente acontece en los procesos de enseñanza y en la interacción entre docentes y estudiantes en el área de desarrollo de habilidades para resolución de problemas, en términos de la existencia de patrones y actividades típicas de aula. Por este motivo, el objetivo de este estudio es analizar las actividades típicas de aula (ATA) en clases de resolución de problemas matemáticos, con el propósito de identificar el tipo de acciones vinculadas a su enseñanza en el nivel primario. Los objetivos específicos son dos: 1) describir las ayudas pedagógicas y las estructuras de participación presentes en las clases de resolución de problemas matemáticos, y 2) caracterizar el trabajo colaborativo entre estudiantes en las clases de resolución de problemas matemáticos.

El aprendizaje colaborativo es definido como el uso didáctico de equipos reducidos de alumnos, en general de composición heterogénea en rendimiento y capacidad, aunque ocasionalmente pueden ser más homogéneos, utilizando una estructura de la actividad tal que 
asegure al máximo la participación equitativa (para que todos los miembros del equipo tengan las mismas oportunidades de participar) y se potencie al máximo la interacción simultánea entre ellos, con la finalidad de que todos los miembros de un equipo aprendan los contenidos escolares (Pujolás, 2009).

La cooperación consiste en trabajar juntos para alcanzar objetivos comunes. En una situación cooperativa, los individuos procuran obtener resultados que sean beneficiosos para ellos mismos y para todos los demás miembros del grupo. El aprendizaje cooperativo es el empleo didáctico de grupos reducidos en los que los alumnos trabajan juntos para optimizar su propio aprendizaje y el de los demás (Johnson y Johnson, 1999). En esta investigación es preciso considerar y caracterizar el trabajo colaborativo que se da en las clases de matemática.

\section{Método}

La presente investigación responde a una metodología cuantitativa, en la cual se aplica una pauta de evaluación sobre actividades típicas de aula, con el propósito de caracterizar 23 clases impartidas por profesores de matemáticas del sistema escolar chileno. En cuanto a los participantes, estos corresponden a una muestra intencionada de 23 profesores de escuelas municipales evaluadas en las clases de matemáticas entre los niveles $5^{\circ}$ y $8^{\circ}$ de educación primaria, es decir, con alumnos entre 10 y 13 años, en el proceso de evaluación docente en diversas comunas del país. Las clases corresponden a escuelas de zonas céntricas (Región Metropolitana, región de Valparaíso y Región de O'Higgins).

En cuanto a este proceso, se encuentran los registros audiovisuales a disposición, que fueron solicitados conforme la reglamentación de 2013 del Centro de Perfeccionamiento, Experimentación e Investigaciones Pedagógicas (CPEIP), respetando los constructos éticos. Se trabajó con la transcripción de los registros audiovisuales centrando el análisis en el contenido del discurso tanto de profesores como de los alumnos.

\section{Instrumento}

Del análisis de las clases, surgen de manera recurrente determinados patrones que fueron agrupados teniendo a la base el sistema de análisis de Sánchez (2010). Estos patrones fueron organizados en cuatro ámbitos. El primero, referido a los momentos o ciclos que acontecen en las clases, que en este caso en particular corresponden a inicio, desarrollo y cierre. En un segundo ámbito encontramos patrones referidos a actividades típicas de aula (ATA); para efectos de este estudio, estas ATA fueron agrupadas en 11 tipos (véase tabla 1), considerando las que surgen desde el inicio hasta el cierre de la clase. En un tercer ámbito, nos focalizamos en el tipo de estructura de participación que se establece en el aula, las cuales, siguiendo los aportes de algunas investigaciones (Cazden, 1988; Reznitskaya y Anderson, 2006), agrupamos en cinco tipos (véase tabla 2). Finalmente, revisamos las ayudas pedagógicas (véase tabla 3), y la presencia y 
forma en que se daba el trabajo colaborativo (véase tabla 4). Los problemas matemáticos presentes en las clases fueron mayormente de tipo aritmético (uso de una o más operaciones) y de tipo geométrico (uso de figuras y fórmulas). No se encontraron problemas sobre porcentajes y probabilidades.

Tabla 1. Caracterización y definición de las actividades típicas de aula

\begin{tabular}{|c|c|c|}
\hline Momento & Actividad típica de aula & Definición operativa \\
\hline \multirow{4}{*}{ Inicio } & Objetivo de la clase & $\begin{array}{l}\text { Presentación explícita de los propósitos o aprendiza- } \\
\text { jes que se espera lograr en la clase y que por lo gene- } \\
\text { ral forman parte de un todo mayor que es la unidad } \\
\text { didáctica. }\end{array}$ \\
\hline & Conocimientos previos & $\begin{array}{l}\text { Lo que los alumnos saben acerca del tema de la cla- } \\
\text { se, en este caso, acerca de la resolución de problema } \\
\text { y/o de la situación problemática que se plantea. }\end{array}$ \\
\hline & Metodología & $\begin{array}{l}\text { Las indicaciones que se refieren a la forma de trabajo } \\
\text { durante la clase. }\end{array}$ \\
\hline & Indaga & $\begin{array}{l}\text { Los intercambios en términos de preguntas acerca de } \\
\text { un tema, en este caso, el tema vinculado a la resolu- } \\
\text { ción de problema y/o a la situación problemática. }\end{array}$ \\
\hline \multirow{5}{*}{ Desarrollo } & Presentación Problema & $\begin{array}{l}\text { Explicitación de la situación problemática en su } \\
\text { conjunto, considerando la situación planteada y la } \\
\text { información proporcionada. }\end{array}$ \\
\hline & Análisis del problema & $\begin{array}{l}\text { Las acciones vinculadas a la identificación de la infor- } \\
\text { mación proporcionada y su relación con la situación } \\
\text { problemática. }\end{array}$ \\
\hline & Ejecución del problema & $\begin{array}{l}\text { Llevar a cabo las acciones necesarias para la resolu- } \\
\text { ción del problema, entre ellas, lectura del problema, } \\
\text { selección de estrategia para enfrentarlo, selección de la } \\
\text { información, organización de la información, selección } \\
\text { de operación, ejecución y organización de la respues- } \\
\text { ta. }\end{array}$ \\
\hline & Comprobación del problema & $\begin{array}{l}\text { Acciones tendientes a verificar los resultados obteni- } \\
\text { dos que dan respuesta a la resolución del problema. }\end{array}$ \\
\hline & Argumentación & $\begin{array}{l}\text { Fundamentar las acciones y decisiones tomadas } \\
\text { para la resolución del problema. }\end{array}$ \\
\hline \multirow[t]{2}{*}{ Cierre } & Integra & $\begin{array}{l}\text { Reunir toda la información vinculada al problema y } \\
\text { dar la visión de un todo. }\end{array}$ \\
\hline & Valora & $\begin{array}{l}\text { Explicitar la importancia que tienen para el aprendizaje, } \\
\text { las acciones vinculadas a la situación problemática y/o a } \\
\text { la resolución de problemas. }\end{array}$ \\
\hline
\end{tabular}

Elaboración propia. 
Tabla 2. Caracterización de las estructuras de participación

\begin{tabular}{ll}
\hline Estructuras de participación & Definición \\
\hline Monologal & $\begin{array}{l}\text { Habla exclusivamente del profesor. } \\
\text { Ciclos evaluativos o de respuesta única, habitualmente se caracte- } \\
\text { rizan por el inicio del profesor, respuesta estudiantes, evaluación } \\
\text { IRE } \\
\text { Vafesor. } \\
\text { Variante del IRE en que el profesor retroalimenta la respuesta del } \\
\text { estudiante, permitiendo nuevas elaboraciones. }\end{array}$ \\
Frustrol & $\begin{array}{l}\text { Dirigido a controlar el comportamiento en el aula. } \\
\text { Se rompe la interacción discursiva y el profesor no cierra el ciclo. }\end{array}$ \\
\hline & Basado en Sánchez (2010).
\end{tabular}

Tabla 3. Ayudas pedagógicas en las clases de resolución de problemas

\begin{tabular}{ll}
\hline Ayuda pedagógica & Definición \\
\hline Confirma & Corrobora la información que el alumno ya maneja o posee. \\
Corrige & Explicita el error en el alumno. \\
Ejemplifica & $\begin{array}{l}\text { llustra con una situación cotidiana la situación que el alumno no com- } \\
\text { prende. }\end{array}$ \\
Focaliza & Fija la atención del alumno sobre un punto central del problema. \\
Recapitula & Resume al final lo trabajado en la clase. \\
Repite & Vuelve a dar una instrucción o una enseñanza específica. \\
Reformula & Vuelve a dar una instrucción, pero con otras palabras. \\
Completa & Da por sentada la solución, a la espera que el alumno termine la solu- \\
& ción y/o expresión. \\
Motiva & Hace que el alumno manifieste interés por el problema planteado. \\
Trasferencia & Lleva el contenido a una nueva situación, sea cotidiana o teórica. \\
\hline
\end{tabular}

Elaboración propia.

Tabla 4. Tipo de trabajo colaborativo en la sala de clases

\begin{tabular}{ll}
\hline Tipo de trabajo grupal en la sala de clases & Definición \\
\hline Trabajo en parejas & $\begin{array}{l}\text { Trabajo que se da en clases por indicación del } \\
\text { profesor, pero no necesariamente implica una in- } \\
\text { teracción pedagógica. }\end{array}$ \\
& $\begin{array}{l}\text { Los estudiantes trabajan en grupos, pero sin ma- } \\
\text { Interdependencia positiva bien definida }\end{array}$ \\
& $\begin{array}{l}\text { Los estudiantes trabajan en grupos y el profesor } \\
\text { se acerca a cada uno de los estudiantes durante } \\
\text { la clase. } \\
\text { Responsa interacción fomentadora }\end{array}$ \\
& Los estudiantes trabajan en grupos con roles pre- \\
& viamente definidos.
\end{tabular}

Fuente: información extraída de Espinosa (2002). 


\section{Análisis de la información}

En primer lugar, se llevó a cabo un análisis descriptivo mediante porcentajes para determinar la presencia de las distintas acciones (ATA, estructuras, ayudas, colaboraciones) en las fases en que se dividió la clase. Se acompañó ese análisis descriptivo con una caracterización gráfica comparativa para determinar la presencia de posibles asociaciones entre acciones y elementos.

El análisis de correspondencias múltiples (ACM) es un procedimiento descriptivo de asociación entre variables, entre las modalidades de una misma variable o entre modalidades de variables distintas. No se utilizan contrastes de asociación de significación estadística. En vez de ello se usan aproximaciones gráficas, llamadas mapas perceptuales, con puntos que representan las categorías de las variables y que permiten evaluar visualmente la distancia entre ellas.

El ACM implica construir una tabla de contingencia conformada por $i$ filas y $j$ columnas, lo que da lugar a una tabla I $x \mathrm{~J}$. A partir de la tabla, que contiene las frecuencias conjuntas de las variables y su modalidad, se representa en un espacio factorial bidimensional, la distancia entre modalidades o categorías de las variables consideradas, en el análisis, asumiendo que menor distancia implica asociación entre categorías y mayor distancia lo contrario (Vivanco, 1999).

El espacio factorial se construye por ejes o planos de proyección, tomados de dos en dos, de un conjunto de factores que han sido obtenidos en la solución preliminar del análisis. La elección de los ejes, en los que se proyectan las variables y/o sus categorías, está fundada en criterios objetivos y subjetivos.

Una vez elegidos los ejes factoriales o dimensiones, se proyecta en ellos tanto las observaciones como las modalidades o categorías de cada una de las variables. En dicha situación será necesario estudiar qué tanto contribuye cada objeto o modalidad a la variabilidad del eje y qué tan bien están representados los objetos y/o modalidades por dichos ejes.

La interpretación de los resultados se hace mediante aproximación gráfica, observando el mapa perceptual construido, sin perjuicio de complementar dicha interpretación con los resultados cuantitativos que acompañan el análisis.

En el presente estudio se utiliza la aproximación actualizada de Greenacre (2008) y sus correspondientes procedimientos analíticos, implementados en el paquete de análisis FactoMineR (Lê, Josse y Husson, 2008).

El primer paso consiste en seleccionar las variables que pueden estar asociadas y, en consecuencia, pueden contribuir con mayor fuerza a generar dimensiones con las que se construye un espacio factorial en donde se posicionan las variables categóricas y sus modalidades. Estas ya han sido presentadas previamente.

El segundo paso consiste en aplicar la técnica considerando el número de ejes sobre los cuales se proyectan las categorías para obtener su posición en el mapa perceptual. Normalmen- 
te este criterio no es otro que mantener los ejes que explican la mayor proporción de varianza en los datos. Suelen ser los dos primeros ejes extraídos.

\section{Resultados}

Análisis descriptivo

El primer paso en el proceso de análisis fue obtener una representación porcentual de la presencia de las actividades típicas de aula (ATA), estructuras de participación, ayudas pedagógicas y formas de trabajo colaborativo por cada una de las etapas en las que se dividió la clase. Son 30 acciones por cada una de las 23 clases que eventualmente podrían aparecer en cualquiera de las etapas. La tabla 5 dispone los resultados porcentuales por columna y posteriormente, por fila. 
Tabla 5. Porcentaje columna y fila de las ATA, Estructura, Ayudas y Colaboración según etapas

\begin{tabular}{|c|c|c|c|c|c|c|c|}
\hline \multirow{3}{*}{ ATA } & \multicolumn{3}{|c|}{ Etapas (\% por columna) } & \multicolumn{3}{|c|}{ Etapas (\% por fila) } & \multirow[b]{2}{*}{ Total } \\
\hline & Inicio & & Cierre & Inicio & esarrollo & Cíerre & \\
\hline & & & & & & & \\
\hline Objetivo & 4.7 & 0.2 & 1.0 & 70.6 & 11.8 & 17.6 & 100 \\
\hline Cprevio & 11.8 & 0.1 & 0.2 & $95 \cdot 3$ & 3.1 & 1.6 & 100 \\
\hline Método & $9 \cdot 5$ & 2.3 & 3.0 & 39.8 & $45 \cdot 5$ & 14.6 & 100 \\
\hline Indaga & 12.8 & 16.1 & 11.0 & 12.5 & $75 \cdot 0$ & 12.5 & 100 \\
\hline PresProblema & 0.6 & $4 \cdot 3$ & 1.0 & 2.6 & 92.1 & $5 \cdot 3$ & 100 \\
\hline Análisis & 0.0 & $4 \cdot 5$ & 1.3 & 0.0 & 93.2 & 6.8 & 100 \\
\hline Ejecución & 0.2 & $5 \cdot 7$ & 2.5 & 0.6 & 89.7 & 9.6 & 100 \\
\hline Comprob & 0.0 & 1.9 & 1.0 & 0.0 & 88.5 & 11.5 & 100 \\
\hline Argumenta & 0.0 & 1.6 & 1.3 & 0.0 & 83.0 & 17.0 & 100 \\
\hline Integra & 0.6 & 0.1 & 2.8 & 13.6 & 9.1 & $77 \cdot 3$ & 100 \\
\hline Valora & 0.2 & 0.1 & 2.8 & 4.8 & $14 \cdot 3$ & 81.0 & 100 \\
\hline \multicolumn{8}{|c|}{$\begin{array}{l}\text { Estructura de partici- } \\
\text { pación }\end{array}$} \\
\hline Monologal & 3.9 & 2.4 & 0.8 & 24.1 & 69.9 & 6.0 & 100 \\
\hline IRE & 17.2 & 18.0 & $14 \cdot 3$ & 14.4 & 71.6 & $13 \cdot 9$ & 100 \\
\hline IRF & $3 \cdot 9$ & $3 \cdot 5$ & 3.2 & $15 \cdot 9$ & 69.0 & 15.1 & 100 \\
\hline Control & $4 \cdot 3$ & 8.1 & 26.0 & 5.8 & 52.9 & 41.3 & 100 \\
\hline Frustrado & 2.7 & 2.2 & 1.8 & 17.9 & 67.9 & 14.1 & 100 \\
\hline \multicolumn{8}{|l|}{ Ayudas } \\
\hline Confirma & 8.1 & 8.3 & 5.8 & 14.9 & 72.7 & 12.4 & 100 \\
\hline Corrige & 1.4 & 2.9 & $3 \cdot 3$ & 7.1 & 72.4 & 20.4 & 100 \\
\hline Ejemplifica & 3.9 & 2.7 & 3.8 & 18.3 & 60.6 & 21.1 & 100 \\
\hline Focaliza & 2.7 & 3.1 & 0.5 & 15.1 & 81.7 & 3.2 & 100 \\
\hline Recapitula & 0.6 & 0.4 & 1.7 & 13.6 & 40.9 & $45 \cdot 5$ & 100 \\
\hline Repite & 2.1 & 1.6 & 1.5 & 18.6 & 66.1 & $15 \cdot 3$ & 100 \\
\hline Reformula & 1.4 & 1.2 & 0.5 & 17.9 & 74.4 & 7.7 & 100 \\
\hline Completa & $5 \cdot 4$ & 3.8 & 2.3 & 20.6 & 69.1 & 10.3 & 100 \\
\hline Motiva & 0.6 & 2.2 & 3.8 & 3.7 & 67.9 & 28.4 & 100 \\
\hline Trasfiere & 0.8 & 0.2 & 2.0 & 18.2 & $27 \cdot 3$ & $54 \cdot 5$ & 100 \\
\hline \multicolumn{8}{|c|}{ Trabajo colaborativo } \\
\hline Parejas & 0.0 & 0.2 & 0.0 & 0.0 & 100.0 & 0.0 & 100 \\
\hline Interdependencia & 0.2 & 0.7 & 0.5 & 5.0 & 80.0 & 15.0 & 100 \\
\hline Ext. Interaccion & 0.4 & 1.6 & 0.0 & 4.8 & 95.2 & 0.0 & 100 \\
\hline Individual & 0.2 & 0.2 & 0.2 & 12.5 & 75.0 & 12.5 & 100 \\
\hline Total & 100 & 100 & 100 & & & & \\
\hline
\end{tabular}

En cuanto a los momentos de la clase, se aprecia que en los porcentajes-columna, las acciones más frecuentes durante el inicio de la clase son la activación de conocimientos previos e indagar; en cuanto a las estructuras de participación, es IRE la acción que más se presenta; y en 
cuanto a las ayudas pedagógicas, la más frecuente es la facultad que tiene el profesor de confirmar las respuestas de los estudiantes. Sobre el desarrollo de la clase y según las actividades típicas de aula, las acciones de indaga y ejecución aparecen como las más frecuentes. En cuanto a las estructuras de participación, dominan el IRE y el control. Y en relación con las ayudas pedagógicas, completar y confirmar se evidencian como las principales prácticas en el aula. Y en relación con el cierre de la clase, se mantiene la tendencia antes señalada. Sin embargo, predomina la estructura de control como una acción constante en este momento de la clase.

Considerando los porcentajes-fila por cada uno de los momentos de la clase, también se da una tendencia similar a la mencionada en los resultados por porcentaje-columna. No obstante, destacan en las actividades típicas de aula, el abordaje de la clase misma de resolución de problemas focalizada en presentar el problema, analizarlo, ejecutarlo, comprobarlo y argumentarlo, aunque conviene añadir que estas últimas tres acciones son menores en relación a las dos primeras. En relación con las estructuras de participación, destaca la estructura Frustrado en el desarrollo de la clase, es decir, durante el desarrollo generalmente se rompen los patrones interactivos. Y en cuanto a las ayudas pedagógicas, Motivar es una acción que se da de forma recurrente, generalmente en el desarrollo de la clase.

En los porcentajes-columnas, las ATA Conocimiento previo, Método e Indaga, y la Estructura IRE son las que más aparecen al inicio de la clase. A su vez, Indaga vuelve a aparecer con un porcentaje de importancia en la etapa del desarrollo de clase, así como IRE, IRF, Control y Confirma. En cuanto a la etapa de cierre, las ATA con mayor presencia son Indaga y las estructuras de participación IRE y también, fuertemente, Control.

Los porcentajes-fila, por su parte, además de corroborar las presencias que se han mencionado por cada una de las ATA ya comentadas, también permiten establecer, independiente de su contribución general por etapa, la distribución de las acciones entre etapas. La aparición de la mayoría de ellas se concentra en una fase específica de la clase, mientras que algunas como Método, Recapitula o Trasfiere, además de aparecer poco en términos netos, se distribuyen más uniformemente entre dos o tres fases y no es fácil establecer, en este caso, si son acciones propias de una etapa o no.

Una ayuda a la interpretación proviene del gráfico de balones, en donde a mayor tamaño del balón, mayor presencia de dicha acción en la etapa correspondiente.

Diáloqos 
Figura 1. Gráfico de balones por etapas, para las ATA, estructura, ayudas y colaboración

Etapas*ATAS
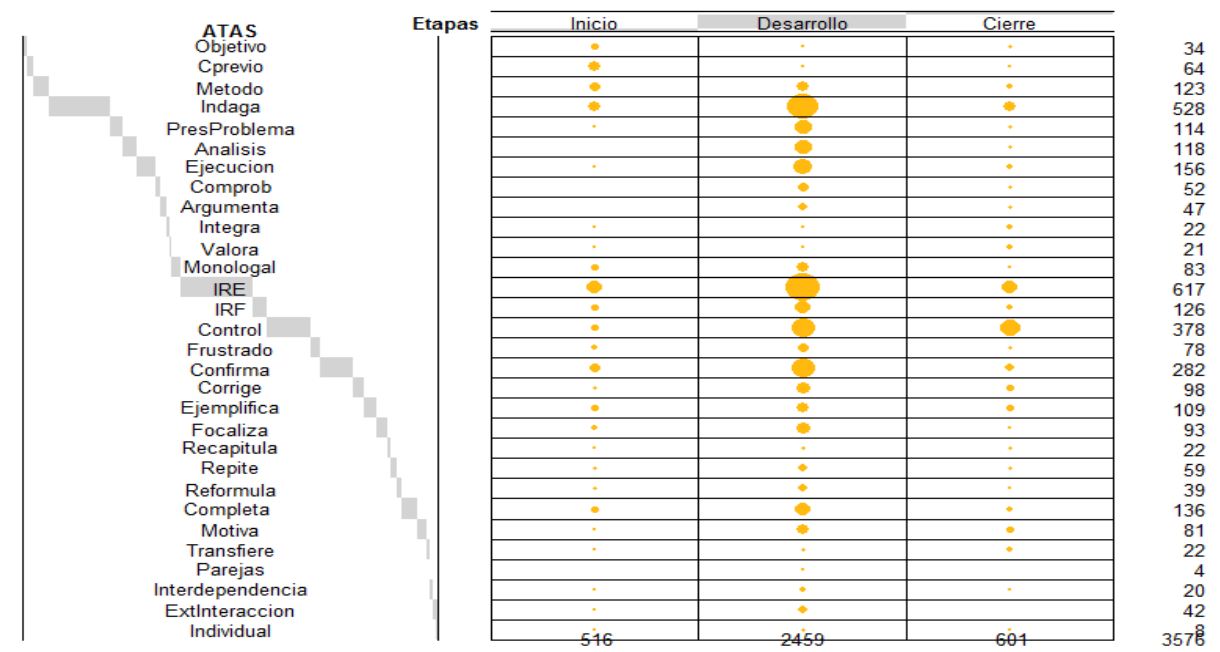

La suma de frecuencias de las columnas corrobora que la mayor presencia de acciones se observa durante la etapa de desarrollo. Esto es esperable pues es el momento de la clase que consume mayor cantidad de tiempo y es donde los docentes llevan a cabo los objetivos y la metodología propuestos en la etapa de planificación. No obstante, es posible establecer alguna asociación entre acciones específicas y fases concretas.

En primer lugar, parece ser que Objetivo, Conocimiento previo y Método son más propias de la etapa de inicio. Aunque sus frecuencias son bajas, denotan mayor presencia que el resto de las acciones.

En segundo lugar, se observa que las estructuras IRE, IRF, Control y Frustrado, así como las ayudas Confirma, Corrige, Ejemplifica y Focaliza, Completa y Motiva, además de evidenciar las mayores frecuencias de aparición - a juzgar por las frecuencias informadas en filas-, están aparentemente vinculadas a la fase de desarrollo de la clase. Llama la atención en este momento de la clase que las acciones Comprobar y Argumentar tienen menos presencia que las acciones Analizar y Ejecutar, siendo que todas ellas deberían estar presente en el abordaje de la RP. Es necesario aclarar que Comprobar y Argumentar son acciones de alta complejidad cognitiva para los estudiantes y, por lo tanto, más desafiantes.

En tercer y último lugar, en la etapa de cierre no parece haber acciones exclusivas o predominantes; sin embargo, se puede destacar mayor presencia de Indaga, IRE y especialmente Control. Aquí también resulta llamativo la baja presencia de las ayudas Trasferencia e Integrar, siendo acciones que debieran ser más notorias en el cierre de la clase dado su carácter de asociación con otros contenidos o aprendizajes. 
Esta descripción preliminar permite descartar algunas de ellas, que virtualmente no figuran en el estudio. Se dejan fuera del ulterior análisis a Objetivo, Conocimiento previo, Integra, Valora, Monologal, Recapitula, Repite, Reformula, Trasfiere, Parejas, Interdependencia, Extinteracción, Individual.

\section{Análisis de asociación}

Para aproximar una mejor interpretación en términos de asociación entre acciones y etapas, se obtiene una primera aproximación mediante el gráfico de mosaico. El resultado se muestra en la figura 2.

Figura 2. Gráfico de mosaico de la asociación entre acciones y etapas

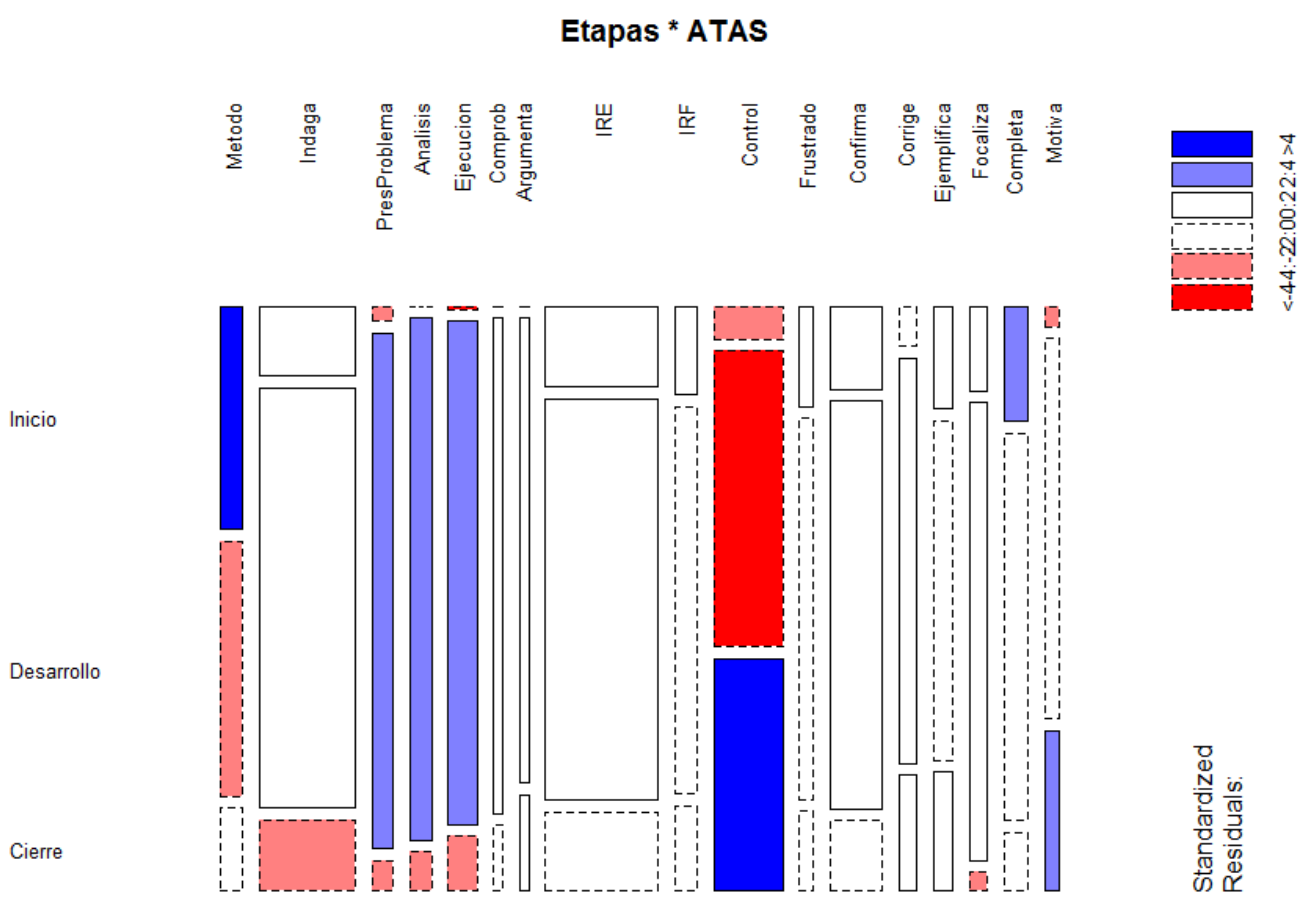

El gráfico muestra barras cruzadas entre etapas (eje Y) y ATA, Estructura y Ayudas (eje X), que representan su mayor o menor frecuencia al cruzar ambas categorías. Así, por ejemplo, al considerar Método, esta aparece con la misma relevancia durante la etapa de Inicio y DesarroIlo, bastante menor en cierre. El color, cuando es azul, indica una frecuencia sobre la esperada, mientras que el color rojo refleja una frecuencia debajo de la esperada. En el caso de Método, su frecuencia es relevante en las etapas mencionadas, sobre lo esperado en el Inicio y bajo lo esperado en Desarrollo. Con esta norma, la lectura de las restantes categorías cruzadas es simple. 
Presentación del Problema, Análisis y Ejecución denotan una presencia arriba de la esperada en el desarrollo de la clase y debajo de lo esperado en inicio y cierre. Control se presenta abajo de lo esperado en inicio y desarrollo, pero sobre lo esperado en cierre. Y Completa, así como Motiva, están encima de lo esperado en inicio y cierre, respectivamente.

Al igual que en los datos anteriores, llama la atención la poca presencia de la Comprobación y la Argumentación durante el abordaje de la RP en el desarrollo de la clase. También resulta clave identificar que el IRF no juega un rol importante durante las clases y la poca presencia de la motivación durante los inicios de las mismas.

Para finalizar, se dispone el análisis mediante correspondencias simples. Se ha obtenido mediante una tabla de contingencia cruzada y los resultados se disponen a continuación:

Tabla 6. Coordenadas, contribuciones y cosenos cuadrados de las acciones por etapas

\begin{tabular}{|c|c|c|c|c|c|c|}
\hline & \multicolumn{2}{|c|}{ Coordenadas } & \multicolumn{2}{|c|}{ Contribución } & \multicolumn{2}{|c|}{$\operatorname{Cos}^{2}$} \\
\hline Etapas & Dimensión 1 & Dimensión 2 & Dimensión 1 & Dimensión 2 & Dimensión 1 & Dimensión 2 \\
\hline Inicio & -0.19 & 0.64 & 5.78 & 82.16 & 0.08 & 0.92 \\
\hline Desarrollo & -0.11 & -0.12 & 11.44 & 17.10 & 0.45 & 0.55 \\
\hline Cierre & 0.61 & 0.05 & 82.78 & 0.74 & 0.99 & 0.01 \\
\hline \multicolumn{7}{|l|}{ ATA } \\
\hline Método & -0.13 & 0.85 & 0.89 & 46.44 & 0.02 & 0.98 \\
\hline Indaga & -0.11 & -0.01 & 2.56 & 0.05 & 0.98 & 0.02 \\
\hline PresProblema & -0.27 & -0.37 & $3 \cdot 52$ & 8.22 & 0.34 & 0.66 \\
\hline Análisis & -0.22 & -0.44 & 2.47 & 12.08 & 0.20 & 0.80 \\
\hline Ejecución & -0.15 & -0.40 & 1.46 & 13.22 & 0.12 & 0.88 \\
\hline Comprob & -0.10 & -0.41 & 0.20 & 4.55 & 0.05 & 0.95 \\
\hline Argumenta & 0.05 & -0.37 & 0.05 & $3 \cdot 37$ & 0.02 & 0.98 \\
\hline IRE & -0.07 & 0.06 & 1.45 & 1.00 & 0.64 & 0.36 \\
\hline IRF & -0.05 & 0.11 & 0.13 & 0.78 & 0.17 & 0.83 \\
\hline Control & 0.67 & -0.02 & 73.17 & 0.08 & 1.00 & 0.00 \\
\hline Frustrado & -0.08 & 0.17 & 0.21 & 1.13 & 0.19 & 0.81 \\
\hline Confirma & -0.12 & 0.06 & 1.62 & 0.52 & 0.79 & 0.21 \\
\hline Corrige & 0.12 & -0.12 & 0.58 & 0.80 & 0.47 & 0.53 \\
\hline Ejemplifica & 0.10 & 0.23 & 0.50 & 2.96 & 0.17 & 0.83 \\
\hline Focaliza & -0.36 & 0.00 & 5.13 & 0.00 & 1.00 & 0.00 \\
\hline Completa & -0.19 & 0.22 & 2.07 & 3.49 & 0.42 & 0.58 \\
\hline Motiva & 0.34 & -0.18 & 3.98 & 1.31 & 0.79 & 0.21 \\
\hline
\end{tabular}

La tabla 6 es una síntesis de la posición, contribución y representación de las acciones listadas y de las tres etapas en las que se divide la clase dentro de un espacio factorial compuesto de dos ejes o dimensiones. La finalidad es establecer asociaciones entre estos elementos. 
Las coordenadas indican la posición de cada categoría en la dimensión 1 y 2, los valores de coordenadas elevados indican lejanía del punto central del espacio factorial y mayor contribución con la varianza de dicho espacio. Las contribuciones representan el aporte en porcentaje de una categoría a la variabilidad de una dimensión -en este caso separadas por variable, acciones por un lado y etapas, por otro. Por su parte, los cosenos cuadrados indican la calidad de representación de las categorías sobre las dimensiones, los valores cercanos a 1 indican mejor calidad.

Existe una cierta concordancia entre categorías con mayores valores en coordenadas, su porcentaje de contribución y su calidad de representación. Así, entre las etapas de la clase, la dimensión 2 representa mejor el inicio y el desarrollo, mientras que la dimensión 1 lo hace mejor con la etapa de cierre. Entre las acciones, las mejor representadas y que más contribuyen a la dimensión 2 son Método, Análisis y Ejecución. En la dimensión 1 contribuyen más las acciones Control y Figurando, pero en mucho menor medida, la acción Focaliza.

Se puede tomar esta información preliminar para interpretar el mapa perceptual que se dispone a continuación, observando particularmente aquellas etapas y acciones mejor representadas.

Figura 3. Mapa perceptual de acciones y etapas de la clase

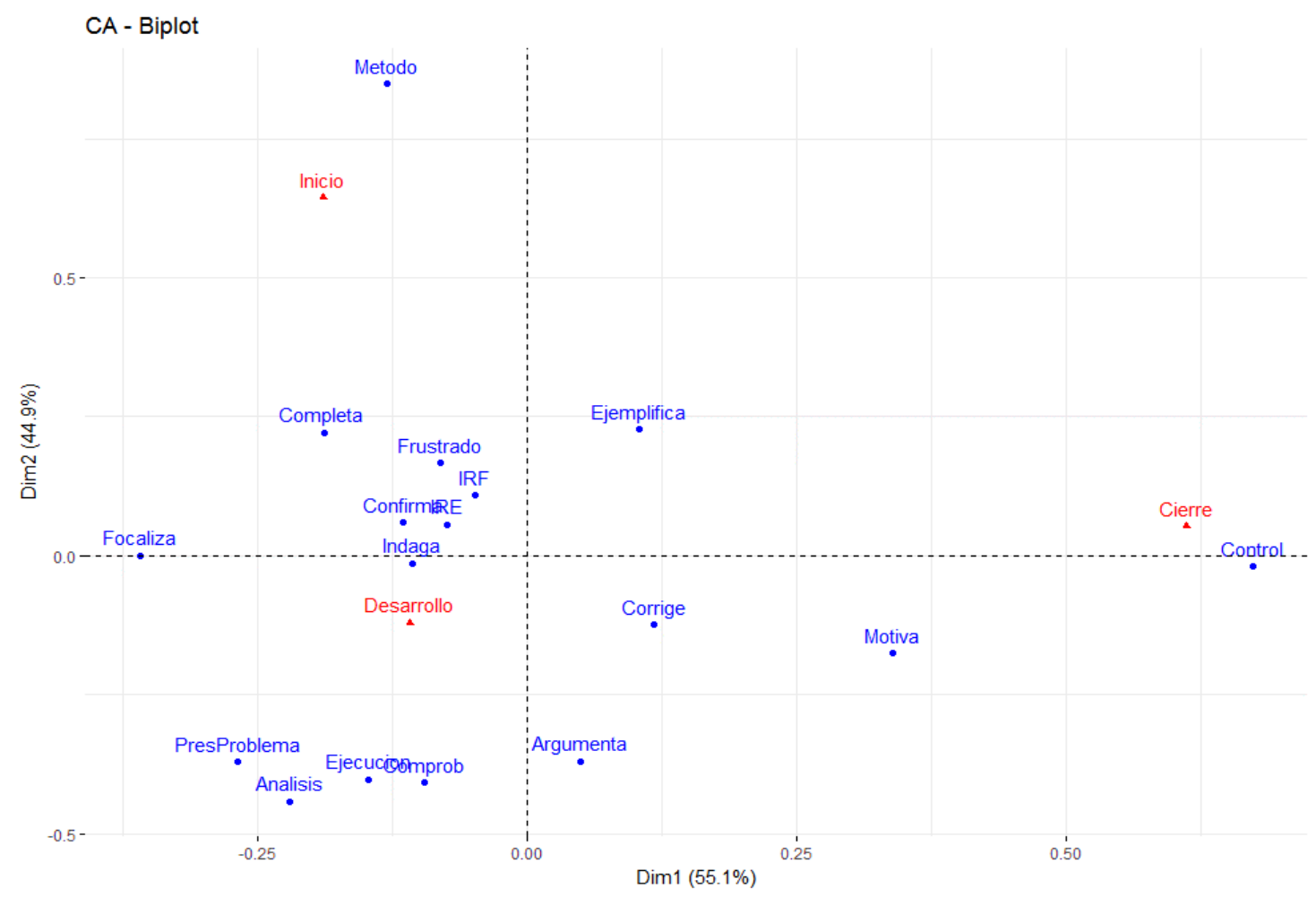

Diálǫos 
Dado que es un espacio factorial propio de un análisis de correspondencias simples, la proporción de varianza explicada llega a 100\%. La dimensión 1 explica 55\% de dicha varianza, mientras que la dimensión 2 explica 45\%.

Todas las etapas de clase están bien representadas y contribuyen de modo relevante a la construcción de las dimensiones. Todas son interpretables. Lo primero que se aprecia es que tanto Inicio como Cierre, se alejan del centro y, en consecuencia, contribuyen más con la varianza del espacio factorial, pero a su vez, son etapas en que las ocurrencias de acciones son claramente menos frecuentes $y$, por tanto, aparecen menos asociadas con otras categorías. Desarrollo, en cambio, representa una fase en la que ocurren acciones con mayor frecuencia.

Por su parte, como se ha evidenciado en la tabla, no todas las acciones contribuyen o están bien representadas en este espacio factorial. Contribuyen más a la varianza y son menos frecuentes, las acciones Método, Análisis, Control, Motiva y Focaliza.

Al estudiar las asociaciones, dadas por proximidad espacial, es claro que la etapa de inicio está asociada fuertemente con Método, aunque ambas, etapa y acción, no son frecuentes dentro del total de acciones evaluadas. Algo similar ocurre con Cierre, fuertemente asociada con la acción Control. Desarrollo y las acciones vinculadas a ella, son bastante frecuentes en el total de acciones contabilizadas. Su mayor asociación se encuentra con Presentación del problema, Análisis, Ejecución y Comprobación. Otras acciones, como IRE e IRF, que antes parecían asociadas, no reflejan una frecuencia mayor o menor a lo esperado respecto de la etapa de asociación, evidenciando además una menor calidad de representación, lo que hace más incierta su calidad de interpretación.

\section{Conclusiones}

El objetivo de este estudio fue analizar las ATA en clases de resolución de problemas matemáticos con el propósito de identificar el tipo de acciones vinculadas a su enseñanza en nivel primario. Los objetivos específicos fueron dos: 1) describir las ayudas pedagógicas y las estructuras de participación presentes en las clases de resolución de problemas matemáticos, y 2) caracterizar el trabajo colaborativo entre estudiantes en las clases de resolución de problemas matemáticos. Para ello se llevó a cabo un análisis de correspondencias múltiples (ACM).

Recordemos que el sistema de análisis permitió identificar 11 ATA (Tabla 1) en las clases de resolución de problemas. Estas ATA se encuentran presentes a lo menos en dos de los tres momentos de la clase. Entre las ATA se destaca la activación del conocimiento previo como una actividad muy recurrente en las clases, en especial en el momento de inicio, mientras que en el desarrollo de la clase, las ATA a las que más se recurre son la presentación del problema, el análisis del mismo y la ejecución del problema. En menor medida aparecieron la comprobación y la argumentación de estas actividades. 
Respecto del objetivo específico 1, en relación con describir las ayudas pedagógicas en las clases de resolución de problemas matemáticos, se concluye que fue posible identificar y describir diez tipos de ayuda que se encuentran presentes principalmente durante el desarrollo de la clase, mientras que las estructuras de participación identificadas fueron cinco, destacándose la presencia de IRE en los momentos de inicio y desarrollo, y de control, en el momento del cierre. Respecto del objetivo específico 2, queda en evidencia la escasez de instancias de trabajo colaborativo en el aula $y$, aunque este se propicie, no se logra el intercambio entre alumnos y entre profesor y alumnos.

Al analizar los procesos que se evidencian en los momentos de la clase se puede relevar que los momentos de inicio y cierre son a los que se destina menor número de acciones en comparación con el desarrollo de la clase, momento en el que ocurren la mayoría de las situaciones. Si bien esto es de esperar por la variable tiempo, hay acciones que no tienen fuerza en los momentos en los que se supone se deben abordar en términos de orientaciones y apoyos a la tarea que se va a desarrollar, el inicio debe tener una orientación destinada a ello. Si bien al inicio de la clase se activa conocimiento previo y se indaga acerca de lo que los alumnos saben del tema vinculado al problema, no se explicita el objetivo de la clase ni la metodología con que se trabajará.

En el inicio de una clase se espera la presentación del objetivo en una clase de resolución de problemas, pues el objetivo puede ser tan diverso como: aprender a resolver un tipo determinado de problema; aprender alguna estrategia para resolver un problema; identificar la estrategia que resulta más pertinente para el problema que se trabajará en clases; representar el problema; identificar datos, o bien, generar otros problemas parecidos al que se presenta en clases, entre otras posibilidades. Igualmente, es importante explicitar o acordar con los estudiantes la metodología de trabajo que se realizará en la clase, de manera que los alumnos cuenten con la información necesaria para comprender qué se espera de ellos.

Por su parte, en el momento de desarrollo de la clase, las actividades de Indaga, Presentación del problema, Análisis, Ejecución y Comprobación se asocian menos de lo esperado que con la etapa de cierre, como la Comprobación y la Argumentación; ambas tareas de gran ayuda para estudiantes. Si pensamos la actividad matemática como una tarea de alta complejidad cognitiva, entonces la comprobación y la argumentación debieran ser tareas centrales en las de matemáticas.

Con respecto al Método, está fuertemente asociado con el inicio. Esto quiere decir que los profesores no recuerdan la metodología durante el desarrollo de la clase, sino que simplemente la asocian al objetivo.

El Control está fuertemente asociado con el cierre. Si pensamos que la estructura de control es una acción de manejo de grupo, podemos inferir que, a medida que avanza la clase, el profesor demanda llevar a cabo esta forma de abordaje de la interacción en el aula. El control 
está presente mucho menos de lo esperado en Inicio y Desarrollo. Diferente a lo que pasa con la motivación, que está más asociada con el cierre de la clase, lo que se asocia a tener solo como objetivo la tarea terminada, sin embargo, se espera que esta acción sea transversal.

Presentación del Problema, Análisis, Ejecución y Comprobación se asocian más de lo esperado con la etapa de desarrollo. Sin embargo, la Comprobación y la Argumentación son acciones menos frecuentes en relación con la Presentación del problema, Análisis y Ejecución. Esto quiere decir que se realizan los problemas propuestos, pero no se comprueban ni menos se incentiva la capacidad argumentativa.

Se evidencian escasas acciones que apunten al trabajo colaborativo. Según la representación porcentual del análisis descriptivo, la principal acción fomentada en clases es el trabajo en parejas sin interacción pedagógica. En el caso de las aulas chilenas, los estudiantes suelen organizarse en filas y en parejas. Esto quiere decir que con la simple instrucción "trabaje con su compañero de puesto" es suficiente para los docentes como instrucción de una forma de abordar la colaboración. Sin embargo, como hemos visto en las páginas anteriores, la cantidad de interacción entre profesor y alumno es mínima y, además, la calidad de esa interacción es pobre. Ocurre lo mismo para el caso de la extensa interacción fomentadora, donde los estudiantes se reúnen en grupos y el profesor se acerca durante la clase. Si bien es una forma más amplia de colaboración, las ayudas y las estructuras siguen siendo mínimas, lo que implica una dificultad relevante para atender las habilidades argumentativas de los estudiantes.

Las ayudas más frecuentes son de bajo impacto en el aprendizaje y en la mediación, se enfocan a aclarar dudas o resolver inquietudes, más que a utilizar estrategias concretas que beneficien el aprendizaje de los estudiantes. Las interacciones entre profesor y alumno no tienden a la retroalimentación, se enfocan en la ejecución de la tarea y no considera un proceso de monitoreo de las acciones ejecutadas en el desarrollo.

Estos resultados comparten los principios de Bravo, Falck, González, Manzi y Peirano (2008) y de Manzi, Strasser, San Martín y Contreras (2008) en cuanto a la relevancia de mirar los aprendizajes en resolución de problemas, dado el impacto que tienen las decisiones docentes para los estudiantes con mayores problemas de aprendizaje. Lo mismo ocurre con Hino (2007), en cuanto a la relevancia del comportamiento de los estudiantes en el aula y en las clases de resolución de problemas. Los resultados de esta investigación permiten una indagación, aunque inicial, en las formas en que se tipifican las decisiones pedagógicas. Por su parte, Lemke (1998) da relevancia a la interacción social que se da entre los docentes y los estudiantes, los cuales - según los resultados parciales de este estudio - son de baja retroalimentación y contacto social. Las proyecciones apuntan a seguir profundizando en esta temática con muestras más robustas y diversos niveles de enseñanza.

A modo de cierre, es necesario señalar que, en la medida que los profesores realicen actividades de mayor complejidad en el aula, hay mayores posibilidades para que los estudiantes 
adquieran estrategias para resolver problemas en conformidad a los propósitos que se espera en el eje de habilidades matemáticas en el curriculum nacional (Mineduc, 2009).

Uno de los desafíos es continuar investigando en la resolución de problemas aplicado a distintas ATA y en diferentes niveles de curso. Esto porque es de interés conocer cuáles son las actividades más frecuentes a las que recurren los profesores cuando enseñan resolución de problemas.

\section{Referencias bibliográficas}

Aguilar Villagrán, M.; E. Aragón Mensizábal; J. I. Navarro Guzmán (2015). Las dificultades de aprendizaje de las matemáticas (DAM). Estado del arte. Revista de Psicología y Educación, $10(2), 13-42$.

Alfaro, L.; R. Gormaz (2009). Análisis comparativo de los resultados chilenos en las pruebas de Matemática SIMCE y PISA. ¿Qué nos dice PISA sobre la educación de los jóvenes en Chile? Nuevos análisis y perspectivas sobre los resultados en PISA 2006. Unidad de Currículos y Evaluación del Mineduc, 239-260. Chile: Gráfica 7.

Alsina, A. (2012). Cómo desarrollar el pensamiento matemático de 0 a 6 años. Barcelona: Ediciones Octaedro.

Alsina, A. y N. Planas (2008). Matemática inclusiva. Propuestas para una educación matemática accesible. Madrid: Ediciones Narcea.

Alvarado, M.; G. Cabezas; D. Falck; M. E. Ortega (2012). Serie evidencias: Evaluación docente y resultados de aprendizaje ¿Qué nos dice la evidencia? Santiago: Mineduc.

Araya, R.; P. Dartnell (2008). Saber pedagógico y conocimiento de la disciplina matemática en profesores de educación general básica. Proyecto FONIDE № 2122006.

Bravo, D.; D. Falck; R. González; J. Manzi; C. Peirano (2008). La relación entre la evaluación docente y el rendimiento de los alumnos: Evidencia para el caso de Chile. Chile. http://eoepsabi.educa.aragon.es/descargas/H Recursos/h 1 Psicol Educacion/h 1.4.Eval desemp docente/1.5.Relacion evaluac doc rendim.pdf

Brissiaud, R.; E. Sander (2005). Arithmetic Word Problem Solving: A Situation Strategy First Framework. Developmental Science, (13), 92-107.

Callejo, M. (1996). Evaluación de procesos y progresos del alumnado en la resolución de problemas. UNO, Revista de Didáctica de las Matemáticas, 53-63.

Chamorro, M. (2006). Didáctica de las matemáticas. Madrid: Pearson.

Cazden, C. B. (1988). Classroom Discourse: The Language of Teaching and Learning. Portsmouth: Heinemann.

Copeland, M. (2004). Socratic Circles: Fostering Critical and Creative Thinking in Middle and High School. Portland: Stenhouse Publishers. 
Espinosa, G. (2002). Trabajo en equipos dentro del aula. Boletin UMC. Perú: Ministerio de Educación.

Espinoza, L.; J. Barbé; G. Gálvez (2009). Estudio de fenómenos didácticos vinculados a la enseñanza de la aritmética en la educación básica chilena. Enseñanza de las Ciencias, 27(2), 157-168.

Felmer, P.; J. Peldormo-Díaz; T. Cisternas; F. Cea; V. Randolph; L. Medel (2014). La resolución de problemas en la matemática escolar y en la formación inicial docente. Informe Fonide. Montevideo: Ministerio de Educación.

García, R.; J. Rosales; E. Sánchez (2010). La lectura en el aula: qué se hace, qué se debe hacer y qué se puede hacer. Barcelona: Graó.

Greenacre, M. (2008). La práctica del análisis de correspondencias. Bilbao: Editorial Fundación BBVA.

Giménez, J.; J. Pedro da Ponte (2004). La actividad matemática en el aula. Homenaje a Paulo Abrantes. Barcelona: editorial Graó.

Hino, K. (2007). Toward the Problem-Centered Classroom: Trends in Mathematical Problem Solving in Japan. ZDM The International Journal on Mathematics Education, 39(5), 503-514.

Johnson, D. W.; R. T. Johnson; E. J. Holubec (1999). El aprendizaje cooperativo en el aula. Buenos Aires: Paidós.

Lara, B.; A. Mizala; A. Repetto (2010). Una mirada a la efectividad de los profesores en Chile. Estudios Públicos, 120, 147-181. Chile: CEP.

Lê, S.; J. Josse; F. Husson (2008). FactoMineR: Un paquete R para análisis multivariante. Revista de Software Estadístico, 25(1), 1-18.

Lemke, J. L. (1998). Multiplying Meaning: Visual and Verbal Semiotics in Scientific Text. Martin, J. R.; R. Veel (eds.). Reading Science. Londres: Routledge, 87-113.

Manzi, J.; K. Strasser; E. San Martín; D. Contreras (2008). Quality of Education in Chile: Final Report of the Interamerican Development Bank Proyect. Washington: BID. http://www.iadb.org/res/ laresnetwork/files/pr300finaldraft.pdf

Ministerio de Educación (2009). Objetivos fundamentales y contenidos mínimos obligatorios de la educación básica y media. Santiago, Chile: Mineduc.

Olfos, R. (2010). Conocimiento pedagógico del contenido y su incidencia en la enseñanza de la matemática a nivel de educación básica. Proyecto FONIDE No F410980. Valparaíso: Pontificia Universidad Católica de Valparaíso. https://centroestudios.mineduc.cl/wp-content/uploads/ sites/100/2017/07/Informe-Final-PUCV-Raimundo-Olfos.pdf

Orrantia, J.; D. Múñez; M. Fernández; L. Matilla (2012). Resolución de problemas aritméticos: Conocimiento conceptual y nivel de competencia en matemáticas. Aula Abierta, 40(3), 23-32. Oviedo: Universidad de Oviedo.

Polya, G. (1965). Cómo resolverlo: Un nuevo aspecto del método matemático. Madrid: Princeton. 
Preiss, D.; A. Larraín; S. Valenzuela (2011). Discurso y pensamiento en el aula matemática chilena. PSYKHE, 20(2), 131-146.

Pujolás, P. (2009). Aprendizaje cooperativo y educación inclusiva. Una forma práctica de aprender junto a alumnos diferentes. VI Jornadas de Cooperación Educativa con Iberoamérica sobre Educación Especial e Inclusión Educativa. Antigua (Guatemala).

Reznitskaya, A.; R. Anderson (2006). Analyzing Argumentation in Rich, Natural Contexts. Informal Logic, 26(2), 175-198.

Rosales, J.; J. Orrantia; S. Vicente; J. M. Chamoso (2008). La resolución de problemas aritméticos en el aula. ¿Qué hacen los profesores cuando trabajan conjuntamente con sus alumnos? Cultura y Educación, 20(4), 423-439.

Sánchez, E. (2003). ¿Realmente somos conscientes de lo que supone alfabetizar? Textos de Didáctica de la Lengua y de la Literatura Barcelona, 9(33), 62-77.

Sánchez, E.; C. Coll (2007). Presentación. El análisis de la interacción alumno-profesor: Líneas de investigación. Revista de Educación, (346), 15-32.

Sánchez, E.; E. García; N. Castellano; R. Sixte; A. Bustos; H. García-Rodicio (2008). Qué, cómo y quién: Tres dimensiones para analizar la práctica educativa. Cultura y Educación, 20(1), 95118.

Sánchez, E.; R. García; J. Rosales (2010). La lectura en el aula. Qué se hace, qué se debe hacer y qué se puede hacer (Colección Crítica y Fundamentos). España: Editorial Graó.

Sánchez, E. (coord.). (2010). La lectura en el aula. España: Editorial Graó.

Sinclair, J.; M. Coulthard (1975). Towards an Analysis of Discourse: The English Used by Teachers and Pupils. Londres: Oxford University Press.

Tambychik, T.; T. Mohd Meerah (2010). Students' Difficulties in Mathematics Problem-Solving: What Do They Say? Procedia - Social and Behavioral Sciences, (8), 142-151. https://doi. org/10.1016/j.sbspro.2010.12.020

Toboso, J. (2005). Evaluación de las habilidades cognitivas en la resolución de problemas matemáticos. (Tesis). España: Universidad de Valencia.

Thevenot, C.; M. Devidal; P. Barrouillet; M. Fayol (2007). Why Does Placing the Question before an Arithmetic Word Problem Improve Performance? A Situation Model Account. Psychology press. Doi:10.1080/17470210600587927

Valdés, R. (2015). Los problemas aritméticos de enunciado verbal, según Luria y Tsvetkova, al finalizar primer ciclo de enseñanza básica en escuelas municipales de la comuna de Talca. Perspectiva Educacional, 54(2), 92-108.

Vivanco, M. (1999). Análisis estadístico multivariable. Santiago: Editorial Universitaria.

Diólopos 